Viewpoint

\title{
Potential of laser-induced ablation for future space applications
}

\author{
Alison Gibbings ${ }^{\mathrm{a}, \mathrm{c}, *}$, Massimiliano Vasile $^{\mathrm{a}}$, John-Mark Hopkins ${ }^{\mathrm{b}}$, David Burns ${ }^{\mathrm{b}}$, Ian Watson ${ }^{\mathrm{c}}$ \\ ${ }^{a}$ Advanced Space Concepts Laboratory, Department of Mechanical and Aerospace Engineering, University of Strathclyde, 75 Montrose Street, Glasgow G1 1X1, UK \\ ${ }^{\mathrm{b}}$ Institute of Photonics, University of Strathclyde, Wolfson Centre, 106 Rottenrow, Glasgow, UK

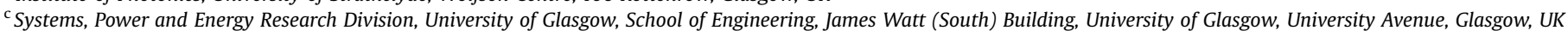

\section{A R T I C L E I N F O}

\section{Article history:}

Received 24 February 2012

Received in revised form

21 May 2012

Accepted 22 May 2012

Available online 16 June 2012

\begin{abstract}
A B S T R A C T
This paper surveys recent and current advancements of laser-induced ablation technology for spacebased applications and discusses ways of bringing such applications to fruition. Laser ablation is achieved by illuminating a given material with a laser light source. The high surface power densities provided by the laser enable the illuminated material to sublimate and ablate. Possible applications include the deflection of Near Earth Objects - asteroids and comets - from an Earth-impacting event, the vaporisation of space structures and debris, the mineral and material extraction of asteroids and/or as an energy source for future propulsion systems. This paper will discuss each application and the technological advancements that are required to make laser-induced ablation a practical process for use within the space arena. Particular improvements include the efficiency of high power lasers, the collimation of the laser beam (including beam quality) and the power conversion process. These key technological improvements are seen as strategic and merit greater political and commercial support.
\end{abstract}

(c) 2012 Elsevier Ltd. All rights reserved.

\section{Introduction}

In the past 30 years the advancement of laser technology has enabled the development of many forms of space-based applications to be considered. These have included long-range wireless communications, power transmission, and LIDAR for remote Earth observation and sensing applications. In this paper a new family of advanced space-based applications will be discussed. These include: launching payloads into low-Earth orbit (LEO); the de-orbiting of space debris; the deflection of Near-Earth Objects (NEOs); and the collection of previously inaccessible extraterrestrial material. Consideration of these techniques is only now possible thanks to the maturation of advanced laser technologies - most notably semiconductor and $\mathrm{Yb}$ :fibre sources in providing a coherent light output. Also the specification and detailed requirements of laser sources for more advanced, spacebased application are now being examined.

Lasers are now widely available in a range of wavelengths and output powers, and allow many advantages over conventional

\footnotetext{
* Corresponding author. Advanced Space Concepts Laboratory, Department of Mechanical and Aerospace Engineering, University of Strathclyde, 75 Montrose Street, Glasgow G1 1X1, UK.

E-mail addresses: alison.gibbings@strath.ac.uk (A. Gibbings), massimiliano.vasile@strath.ac.uk (M. Vasile), johnmark.hopkins@strath.ac.uk (J.-M. Hopkins), d.burns@strath.ac.uk (D. Burns), ian.watson@glasgow.ac.uk (I. Watson).
}

techniques - in many circumstances their use is perhaps the only sensible alternative to such techniques. However, the choice and deployment of lasers will largely be determined by the overriding factors of cost, complexity and, critically, the efficiency of both the laser systems and the method in which the light and matter interaction is utilised. Furthermore, while a laser may perform effectively on Earth, additional design and development is often needed to prepare the system for operations within the harsh space environment. Despite this, various new candidate technologies, such as the dielectric and semiconductor disk laser, have shown considerable promise. This could be used to overcome some of the key deployment criteria.

Recently the potential savings in both spacecraft mass and efficiency have made space scientists, engineers and policy makers readdress the prospects of using solar-powered lasers in space. While the initial concept is not new, it is being reconsidered in light of the countless advancements in laser technology over the past 10 years. In particular, semiconductor-laser-pumped solid state and fibre lasers are more compact, lighter and simpler to operate than their gaseous and chemical equivalents. However, further increases in laser efficiency, increased power output and reduction in size are desirable to achieve laser-based ablation for space-based applications. The development of a space-based laser system is currently seen as strategic and was included within the European Space Agency (ESA) technology roadmap - with considerable and ongoing investment being committed. ESA open tenders have 
recently addressed several aspects of laser technology. These have included direct solar pumping, improved efficiency and improved beam quality. Moreover, the European Commission (EC) has included lasers as a key technological development in the last calls for space, as part of the Framework VII proposal. This clearly demonstrates the persistent interest in pushing laser technology beyond its current boundaries. Nevertheless, before any laser space-based product can be brought to market, considerable effort still remains. This can only be achieved through wise investment and focused research and development.

Within this paper the four main and developing space-based applications are presented. These exploit the use of laser technology and matter interaction. They are launching and controlling spacecraft in space; the de-orbiting of space debris; deflecting, capturing and dissecting NEOs; and extracting and exploring surface and subsurface material.

\section{Launching vehicles into space: propulsion, altitude and station keeping}

Access to space, and in particular to LEO, has been, and is currently dominated by the use of chemically powered engines. These represent an energy inefficient, expensive and polluting form of propulsion. They also often need a significant amount of hardware and the continual storage of fuel. For a given launch vehicle, the thrust is achieved by igniting the propellant carried onboard; therefore the maximum energy is limited by the fuel's chemical composition and mass.

Laser ablation, in theory, and demonstrated at a small scale, could be used to provide an alternative and novel access to LEO, and beyond. Instead of burning propellant, previous proposals have suggested the use of a highly focused or collimated laser beam as a remote energy source $[1,2,32]$. Supplied by either a ground or space-based facility, a laser would be used to transform the exposed material - either a solid or a liquid - directly into a gas. This transformation creates a small and extended plume of ejecta that is considered to be an analogue of the rocket exhaust in standard methods of propulsion. Acting on the surface of the given body, the ejecta plume provides a resultant force that would be used to propel the spacecraft into space. Alternatively thrust can also be achieved by using a laser-induced blast wave $[2,4]$. Here, by illuminating sufficiently dense portions of the atmosphere, a highly focused laser beam can create a series of detonations. Sequentially these detonations, in theory and tested at small scale within the laboratory, can be used to propel the given spacecraft to an altitude of about $30 \mathrm{~km}[2,4,5]$. Beyond this the atmospheric pressure is not sufficiently high to initiate the detonation process, and therefore would have to be combined with a rocket motor [2,5]. The performance of atmospheric detonation can, however, be improved by first containing the spacecraft with a launch tube [6]. Prior to detonation, the inclusion of a dense gas behind the spacecraft can be used to increase the pressure contained within the launch tube. This makes the technique more effective - increasing the impulse and propulsion efficiency - and controllable [6,7]. Both techniques are advantageous as they eliminate the need for the spacecraft to carry a dedicated propulsion system. This saves mass, allowing for a higher thrust-to-weight ratio to be maintained.

More research is required to advance the perceived theoretical advantages into an achievable form of propulsion. This should include optimising the momentum coupling between the laser beam and the spacecraft and the formation of the ejecta plume. The control of the spacecraft's altitude during its ascent must also be considered and controlled. Thrust is not just a matter of intensity, but is also governed by the direction and stability of the ablation event. If the latter two issues are solved, then laser ablation could be used to provide localised altitude control and station keeping manoeuvres. Instead of conventional thrusters, small and lightweight $\mathrm{kW}$ lasers, coupled with a reel of fuel tape, could be integrated into a spacecraft's subsystem. These are known as laser micro plasma thrusters [8]. To date, this novel approach to spacebased object manipulation has been tested within the laboratory environment [8]. In a similar fashion laser ablation-driven microplanes have also been proposed [2,5,7].

\section{De-orbiting of space debris}

Over the last 51 years of space activity some 5800 tonnes of obsolete material have been left to accumulate in both LEO and the geostationary orbit [10]. The density of material in these two orbital regions has reached the limit of the runaway Kessler syndrome, where the rate of debris creation through object-toobject collision and fragmentation exceeds the ambient decay rate of the orbiting structures. This leads to the formation of a debris belt and an environmentally unstable population growth which could render orbits unusable $[3,9,10]$. To stabilise this growth, it has been estimated that between five and ten large-size objects will need to be de-orbited per year [11,12]. The active removal of space debris is therefore considered to be one of the most challenging, yet compelling, problems currently facing the space industry. It demands an immediate solution to avoid the catastrophic loss of vital and expensive space assets. To address this impending risk, the EC has provided continual and substantial investment into the area of space debris. Laser ablation could be a potential solution.

A laser beam could be used to illuminate and ablate the surface of any obsolete structure. This would impart a small de-orbiting force onto the structure that would eventually lead it to re-enter the Earth's atmosphere. The exposed surface of the spacecraft provides the propellant source for the ablation process.

For small fragmented structures, $1-10 \mathrm{~cm}$ in diameter, a groundbased laser, equipped with an integrated acquisition and tracking system from an astronomical class telescope could be used [13,14]. The integration of adaptive optics would be needed to overcome the attenuation of the laser beam caused by atmospheric scattering. Adaptive optics can also be used to improve beam pointing, stability and focusing errors.

De-orbiting larger structures would require the use of a spacebased system. This is beneficial as the laser beam can be operational for an extended period of time, with no or only minor restrictions in viewing angle. A small agile spacecraft, mounted with a $\mathrm{kW}$ space-born laser has been proposed $[15,16]$. Each laser would be pumped either directly or indirectly from the Sun. The spacecraft would rendezvous and fly in formation with the given piece of debris, operating in a fetch and de-orbiting fashion. Once the required de-orbiting manoeuvre is achieved, the spacecraft would move onto the next, only de-orbiting one piece at a time. Alternatively this concept could also be adapted via the use of fuel foams, with a known and very low enthalpy of sublimation. Before the ablation event, foam would be attached to the given piece of debris. The laser beam would then sublimate the foam, and not the debris structure [16]. This would guarantee a minimum level of sublimation and associated thrust.

These techniques are advantageous as they avoid the need to physically dock with the potentially non-cooperative (i.e. tumbling or spinning) and/or poorly known targets. However, the success of these techniques requires the ability to sublimate enough of the debris structural mass, without which the required thrust cannot be achieved. It is also critical that the ablation process does not contribute further to the space debris problem. The effect of ablation of a multi-layer, inhomogeneous material also needs to be 
considered. Experimental work is therefore required to fully validate the concept.

\section{Laser deflection of NEOs}

Laser ablation is also considered to be a promising technique in the deflection of asteroids and other NEOs. Asteroid deflection is still considered to be an ongoing and open problem, where at present no definitive solution has been determined. The EC has recently invested some $€ 5$ million into the deflection of asteroids. This underlines the importance of understanding the current impact threat posed, and how to organise, prepare and implement any mitigation measure [27]. Among the asteroid population there are considered to be between 2000 and 20,000 objects - comets and asteroids - that could potentially impact the Earth [17]. Such an impact could result in a range of consequences: from local extinction, to earthquakes and tsunamis, to the catastrophic destruction of life. Therefore to address the impending asteroid-to-Earth impact risk, numerous methods of asteroid mitigation have been examined. These include kinematic impactors, nuclear detonation, mass drivers, low thrust propulsion, gravity tractors and surface ablation. To assess the performance of each technique, a multicriteria, quantitative comparison was performed by Sanchez \& Colombo et al. [18]. Relative to the achievable miss distance, the mass at launch, the warning time and the current technology readiness level, surface ablation was found to be a theoretically promising technique. A high rate of controllable deflection can be achieved with a relatively low mass into space and a short warning time.

Surface ablation can be achieved by either the collection and focusing of direct solar radiation or with the use of a highly focused or collimated laser beam. By continuously illuminating a small portion of the asteroid's surface, the induced ejecta plume exerts a small force onto the asteroid [19,28,33]. Over extended periods of time this can be used to push the asteroid away from its Earth impacting trajectory. Ablation is therefore considered to be a low thrust approach to asteroid deflection.

Previous proposals using laser ablation for asteroid deflection have considered the use of a large, single spacecraft mounted with a megawatt laser. This would be powered from a nuclear reactor $[20,21]$. However, the legal ramifications of launching and operating a nuclear reactor in space, coupled with the significant design and development of a power source and a highly efficient, highpower laser are considered to be extremely difficult. To address these limitations, an alternative laser ablation mission concept has been proposed. Instead of a large monolithic structure, a swarm of lighter, more adaptable spacecraft, each equipped with identical $\mathrm{kW}$ lasers could be used [30]. By flying in formation with the asteroid, and superimposing each laser beam, the cumulative power density on the surface of the asteroid can be used to initiate the ablation process. This permits the delivery of a much more powerful system. The potential for deflection is therefore dependent on the number of spacecraft located within the vicinity of the asteroid, their combined laser power and the material properties of the asteroid. Laser ablation also eliminates the risk of the spacecraft having to physically land and/or attach itself onto the given body. Fragmentation of the asteroid is also avoided.

Based on a relatively small NEO, with an assumed diameter of $250 \mathrm{~m}$ and a mass of $2.7 \cdot 10^{10} \mathrm{~kg}$, and using a series of $22 \mathrm{~kW}$ lasers, deflection distances of thousands of kilometres can be achieved [24]. As required, more spacecraft could be added or removed from the existing constellation. This eliminates the need to design and develop new spacecraft and eradicates the risk of any single-point failure from occurring. System redundancy is therefore increased [22]. Multiple ablation spots can also be used.
Each laser is proposed to be powered either directly or indirectly by the Sun $[22,29]$. This therefore requires the use of a large primary and a smaller secondary mirror to collect the incoming and freely available solar radiation from the Sun. For direct pumping the solar radiation is collected and concentrated directly onto the laser's gain medium. This approach is currently limited by the mismatch between the wide solar emission band and the relatively narrow absorption band of the laser's gain medium. This results in a low level of energy conversion and overall system efficiency. For indirect pumping, the incoming solar radiation is first focused onto a set of highly efficient solar cells located at the back of the primary mirror. This intermediate step is used to convert the incoming radiation into electrical energy that is then used to power the laser and the remaining parts of the spacecraft. Indirect pumping is supported by continued commercial research and development. It is therefore considered to be a superior method, providing a greater efficiency and technology readiness. Electrically pumped semiconductor lasers have a proven plug-in efficiency of over $80 \%$ and optimally designed solar cells can provided a power-to-electricity conversion of up to $40 \%$ [26]. Both units are also commercially available and relatively inexpensive.

However, any exposed surface, including the steering mirror, solar concentrator, radiators and multi-layer insulation, will become susceptible to the degrading effects of the ejecta plume. Within the vicinity of the ejecta plume it is currently assumed that any ablated particle will immediately re-condense and adhere onto the exposed surface. Degradation is considered to follow the Beer-Lambert-Bouguer law [23]. The continued accumulation of the ejecta will decrease the transmittance and increase the absorbance of the exposed surface. The laser beam is also expected to be attenuated by the ablating ejecta plume. Over a period of time this will degrade the performance of the laser's available power, and affect the endurance, efficiency and lifetime of the mission.

To fully understand and examine the operational and environmental constraints of laser ablation, a series of experiments is ongoing. The Advanced Space Concepts Laboratory and the Institute of Photonics, at the University of Strathclyde, in partnership with the Systems, Power and Energy Research Division, School of Engineering at the University of Glasgow, are currently studying the formation and evolution of the ejecta plume and, more importantly, the ejecta's potential for contamination. Using a $90 \mathrm{~W}$ continuous-wave laser, the ablation response of a number of asteroid analogue target materials is being assessed [24,25]. This includes sandstone, olivine, a highly porous composite mixture and a number of meteorites. Assessment has been made relative to the mass flow rate of the ablated sample, the divergence of the ejecta plume and the deposited characteristics of the ejecta. This has enabled the validation and calibration of the current modelling technique. Assumptions within the model have been confirmed, amended and/or eliminated. These experiments continue to demonstrate the applicability of laser ablation as a justified and effective technique for the deflection of NEOs. This is another step closer to realising the potential of lasers in space.

Another important, open question also includes the endurance and efficiency of the laser system. Within the space environment all units will have to operate over an extended period of time. The durability of the laser is dependent on the effectiveness of the spacecraft's heat rejection system, whereas the mass and lifetime of the thermal control system is governed by the efficiency of the laser. The development of technology is therefore expected to advance in both directions: providing low-mass thermal control systems with long-lasting materials and at the same time highefficiency laser systems operating at high temperatures. 


\section{Planetary mining of surface and subsurface material}

Laser ablation has been also revealed to be a valuable method for the successful extraction and exploration of surface and subsurface material. The physical response of laser ablation is to tunnel into the target material and expel material in the form of an ejecta plume. This results in the extraction of deeply situated and previously inaccessible material that can be used for continual scientific and commercial exploitation or exploration. Remote sensing and in-situ analysis of the plume can be used to characterise the chemical composition and physical nature of the ablated material. Confirmed by microscopic analysis, a number of laser ablation experiments has determined that the ejecta plume is chemically identical to the original pre-ablated source material [24]. However, the absorptive properties - deposited ejecta density and absorptivity - of the ejecta are considerably different.

The ejected material could also be physically collected and/or mined. This could be achieved by the spacecraft passing through the plume, and collecting the ejecta via an externally mounted sticky pad or similar mechanism. Analysis of the collected material could then be performed in-situ or returned to Earth as a sample return mission. This would enable scientists to further characterise the composition, formation and evolution of asteroids, and other rocky bodies.

Asteroids, in theory, could also be used for resource exploration and exploitation. Their pristine environment captures the early formation of the solar system, which includes the formation of highly valuable and very rare metals and minerals. This therefore offers a currently untapped commercial opportunity which could be significantly advanced with the use of lasers. Laser ablation could also be used to either capture a relatively small NEO within an Earth-bound orbit, or to slice a larger asteroid into a number of multiple pieces. These individual segments could then be initially captured and/or mined, allowing for large-scale material extraction to be performed. Asteroids could also be used as a staging post. Extracting their inherent hydrogen and oxygen content would enable manned missions to extend their reach across the Solar System and offer continued life support.

\section{Conclusions}

Improvement in laser technology has the potential to radically advance the range of space-related and space-based applications currently being considered within the academic and industrial arena. In particular this paper has discussed how lasers can be used to de-orbit space debris, deflect asteroids and comets, launch and control spacecraft in space and for future extraterrestrial mining ventures. These are only some of the many different possibilities offered by the advancements in laser technology. Long-distance broadband communication and wireless power transmission could also be realised. However, for these techniques to be successful, improvements in laser beam quality and pointing are required. This is needed to generate collimated beams over an extended distance. There also needs to be a radical improvement in the efficiency of lasers that operate at high power levels. Laser systems delivering power from 1 to $10 \mathrm{~kW}$, with a highly collimated beam, should work over an extended period of time, with a wallplug efficiency of $40-50 \%$, or higher [31]. The high efficiency will reduce the mass of the thermal control system and will be competitive with existing low-thrust engines that can already operate at an efficiency of 60-70\%.

Current efforts to coherently combine multiple beams in fibre lasers also seem to be promising. However, they are still far from the desirable power levels that would be needed for an asteroid deflection mission and for wireless power transmission. Direct solar pumping would be an appealing option to remove the additional power conversion stage, although to be competitive, the current efficiency level for both direct and indirect solar pumping (currently 5-10\%) would need to rise substantially to $\sim 20-30 \%$. This could be achieved by the direct solar pumping of laser diodes, but still requires significant research and development to raise the current technology readiness level. Lasers are also required to operate at higher power levels, over a relatively large frequency spectrum. This is to maximise the coupling with the sublimated material or to provide eye-safe wireless power transmission.

The advances in laser technology for space-based applications is also expected to bolster laser technology for terrestrial applications and vice versa. Such applications might include developments in photovoltaic cells, power conversion, heat rejection and the advanced selection of material. These are fundamental technologies that would provide the next generation of energy sources on Earth and are instrumental to enabling the use of any laser system in space.

Among the possible ideas presented in this paper, there is also the use of disaggregate systems. These are formed from a number of small-scale, simple units. These units can be collectively controlled to reach a common goal. The use of disaggregate systems completely avoids the need to scale-up the required technology. The use of a swarm of lasers for asteroid deflection is moving in this direction, offering a flexible and redundant system. Each laser unit can be used to maximise efficiency without an equal increase in the requirement.

It is clear that the deployment of high-power laser systems and related technologies for space-based applications requires a significant and sustained investment into research and development. This would require political impetus at both a national and international level, in order to ensure reliability and, crucially, economic viability. Power of the payload laser and cost are considered to be paramount to this development process. Power can be achieved by combining many lasers onto any single platform. Multiplexing a flotilla of laser-bearing spacecraft could also be used. Efficiency is also considered to be a critical issue. Efficiency governs the size and form of the power source, the engineering and mass of the overall system, as well as the number of required systems for a given application. Questions remain as to the best form of laser for each space-based application, be it a solar-powered or a solar-pumped laser system(s). This will affect the development, implementation and launch of each laser-based application. More research is therefore required.

The authors believe that laser science has matured sufficiently enough that an exploration of the engineering challenges to achieve the required efficiencies in space-worthy system architectures is now timely. This is particularly relevant to the area of solid-state and semiconductor lasers. To this end, key areas of research investment would include: novel engineering concepts in solid-state and semiconductor materials to advance their efficiency; the direct solar-pumping of semiconductor laser devices; the development of energy scavenging mechanisms to recycle unused solar energy that was either reflected, transmitted or converted to heat within the active laser media; the combination of photovoltaic and directly solar-pumped systems; the investigation of beam-combining techniques to alleviate laser power with only minimal payload consequence; and the development of beam-steering/control technologies to improve beampointing and convergence of multiple laser platforms. The combination of all interested commercial and governmental parties is crucial to address these technical challenges and to avoid the delay or even the disabling of these new and vital space technologies. 


\section{References}

[1] Kantrowitz A. Propulsion to orbit by ground-based lasers. Astronautics and Aeronautics 1972;10(5):74-6.

[2] Phipps C, Birkan M, Bohn W, Eckel HA, Horisawa H, Lippert T, et al. Review: laser-ablation propulsion. Journal of Propulsion and Power 2010;26(4): 710-22.

[3] Liou JC, Johnson N. Instability of the present LEO satellite populations. Advanced Space Research 2008;41:1046-53.

[4] Ageev VP, Barchukov AI, Bunkin FV, Konov VI, Korobeinikov VP, Putjatin BV, et al. Experimental and theoretical modelling of laser propulsion. Acta Astronatica 1980;7:79-90.

[5] Bohn WL. Laser Lightcraft performance. Proceedings of SPIE 3885 2002;48. http://dx.doi.org/10.1117/12.376982.

[6] Sasoh A. Laser-drive in-tube accelerator. Review of Scientific Instruments 2001;72(3):1899-983.

[7] Schall WO, Eckel HA, Bohn WL. Laser propulsion thrusters for space transportation, in laser ablation and its applications. Springer Series in Optical Science 2007; 129:435-54.

[8] Phipps CR, Luke J. Diode laser-driven microthrusters: a new departure for micropropulsion. AIAA Journal 2002;40(2):310-8.

[9] Liou JC, Johnson N. A sensitivity study of the effectiveness of active debris removal in LEO. Acta Astronautica 2009;64:236-43.

[10] Kessler DJ, Cour-Palais BG. Collision frequency of artificial satellites: the creation of a debris belt. Journal of Geophysical Research 1978:83(6):2637-46.

[11] Liou J. A Parametric study on using active debris removal for LEO environment Remediation 61st international Astronautical Congress, Prague, Czech Republic, IAC-10.A6.2.5; 2010.

[12] Lewis HG, Saunders A, Swinerd G, Newland RJ. Effects of thermosphere contraction on remediation of the near-earth space debris environment Journal of Geophysical Research 2011;116(A00H08).

[13] Campbell JW. Project orion: orbital debris removal using ground-based sensors and lasers. NASATM 108522; 1996.

[14] Mason J, Stupl J, Marshall W, Levit C. Orbital debris-debris collision Avoidance. Journal of Space Physics, arXiv:1103.1690v3.

[15] Vasile M, Maddock C. Orbital Debris Removal with Solar Concentrators. IAC10-A6.4. 61st International Astronautical Congress, Prague, Czech Republic, October 2010.

[16] Vasile M, Watson I, Sorel M, Maddock C, Gibbings A light Touch: Removing debris with solar light, Study Proposal Ariadna Study Reference No.: AO/ 1-6411/10/NL/CBi 2010.

[17] Campbell J, Phipps C, Reiily J. The impact Imperative - Laser ablation for deflecting asteroids, Meteoroids and comets from impacting the Earth Conference Proceeding in the international Symposium on Beamed energy propulsion, Huntsville, Alabama 2002
[18] Sanchez JP, Colombo C, Vasile M, Radice G. Multi-criteria comparison among several mitigation Strategies for Dangerous Near Earth objects. Journal of Guidance, Control and Dynamics 2009;32(1):121-42.

[19] Melosh H, Nemchinov IV. In: Gehrels T, editor. Non nuclear strategies for deflecting comets and asteroids in hazards due to comets and asteroids. Tucson: University of Arizona; 1994.

[20] Yoo SM, Song YM, Park SY. Spacecraft formation flying for earth-crossing object deflections using a power limited laser ablation. Advances in Space Research 2009;43(12):1873-89.

[21] Park SY, Mazanek DD. Deflection of earth-crossing asteroids/comets using rendezvous spacecraft and laser ablation. Journal of Astronautical Sciences 2005;53(1):21-37.

[22] Vasile M, Maddock C, Summerer L. Conceptual design of a multi-mirror system for asteroid deflection, 27th international Symposium on space technology and science, 5-12 July, Tsukuba, Japan 2009.

[23] Kahle R, Kuhrt E, Hahn G, Knolenberg J. Physical limits of solar collectors in deflecting earth-threatening asteroids. Advanced Science and Technology 2006;10:256-63.

[24] Gibbings A, Vasile M, Watson I, Hopkins JM, Burns D. Experimental analysis of laser ablated plumes for asteroid deflection and exploitation. Acta Astronautica 2012. AA-D-12-00172.

[25] Gibbings, A, Hopkins JM, Burns D, Vasile M, Watson I. On testing laser ablation Processes for asteroid deflection, Conference Proceeding, IAA Planetary Deference Conference, 9-12th May, Bucharest, Romania, 2011.

[26] Landis GA. Prospects for solar pumping semiconductor lasers. In Laser Power Beaming SPIE Proceedings 1994;2121:58-65.

[27] Harris A. A Global approach to near-earth object impact threat mitigation conference proceeding, IAA Planetary Deference Conference, 9-12th May, Bucharest, Romania, 2011.

[28] Phipps CR, Laser deflection of Near-Earth asteroids and comet Nuclei, Proc. International Conference on Lasers 96, STS Press, McLean, VA pg. 580-587, 1997.

[29] Vasile M, Maddock CA. On the deflection of asteroids with mirrors. Celestial Mechanical Dynamics 2010;107:265-84.

[30] Colombo C, Vasile M, Radice G. Optimal low-thrust trajectories to asteroids through an algorithm based on differential dynamic programming. Celestial Mechanics and Dynamical Astronomy 2009. http://dx.doi.org/10.1007/ s10569-009-9224-3.

[31] Phipps C. Laser applications overview: the state of the art and future trend in the United States, RIKEN Review, No 50 in Focused on Laser Precision Microfabrication (LMP); 2002.

[32] Coppersmith J. The cost of reaching orbit: ground based launch systems. Space Policy 2011;27:77-80.

[33] Melosh JH, Nemchinov IV. Solar asteroid diversion. Nature 1993;366: $21-2$. 